\title{
Positioning Psychiatry as a Leader in Trauma-Informed Care (TIC): the Need for Psychiatry Resident Education
}

\author{
Katherine A. Kosman ${ }^{1,2}$ (D) Nomi C. Levy-Carrick ${ }^{1,2}$ \\ Received: 30 November 2018 / Accepted: 3 January 2019 / Published online: 28 January 2019 \\ (C) Academic Psychiatry 2019
}

Trauma is highly prevalent in patient populations, particularly those encountered by psychiatrists and psychiatry residents. Exposure to traumatic events increases an individual's toxic stress burden and increases the risk of mental health problems and chronic physical disease [1]. Adverse childhood experiences (ACEs), including abuse and neglect, have been linked to an array of medical sequelae, including heart, lung, and liver diseases, obesity, diabetes, depression, substance use, and sexually transmitted infections [1-3]. Adverse experiences also lead to epigenetic cellular and molecular mechanisms that produce changes in gene expression, producing lasting effects in the body as well as alterations in neuronal structure and function [4].

The impact of adverse experiences across the lifespan impact interpersonal dynamics as well as individual health, and there is an emerging appreciation of the convergence of discussions about the impact of trauma on workplace morale and safety, with related concerns about resilience and burnout [5]. Trauma is ubiquitous worldwide, whether from adverse childhood experiences (ACEs), abuse, life events, or disasters. The scope of potentially traumatic events has, moreover, expanded in recent years to include experiences of social determinants of health, from food security to race, gender, and religious bias.

Trauma-informed care (TIC) provides a framework for considering trauma at all levels, including patients, providers, and the overall organizational culture. Psychiatry as a field is uniquely positioned to advance trauma-informed care (TIC) and its implications for patient and clinician engagement. The field combines an understanding of neurobiological underpinnings of the effect of trauma, its consequences, and its sequela, with the psychodynamic and psychotherapeutic engagement

Katherine A. Kosman

kkosman@partners.org

1 Brigham and Women's Hospital, Boston, MA, USA

2 Harvard Medical School, Boston, MA, USA with patients in their trauma narrative. Psychiatry resident education in TIC is essential to train the next generation of psychiatrists who are already interacting with patients and who will continue to shape this role in the future.

\section{Background: Trauma and Trauma-Informed Care}

The Substance Abuse and Mental Health Services Administration (SAMHSA) defines trauma as "an event, series of events, or set of circumstances that is experienced by an individual as physically or emotionally harmful or life threatening and that has lasting adverse effects on the individual's functioning and mental, physical, social, emotional, or spiritual well-being" [6]. The prevalence of trauma is exceedingly high. In the 2011 National Stressful Events Survey answered by 2953 individuals in the USA, $89.7 \%$ of respondents reported exposure to at least one DSM-5 Criterion A event, and 30\% of respondents had experienced six of these event types listed in the survey, including disaster, accident/fire, exposure to hazardous chemicals, combat or warzone exposure, physical or sexual assault, witnessed physical/sexual assault, witnessed dead bodies/parts unexpectedly, threat or injury to family or close friend due to violence/accident/disaster, death of family/close friend due to violence/accident/disaster, or work exposure [7].

The landmark 1998 CDC-Kaiser Permanente Adverse Childhood Experiences (ACE) study by Felitti and Anda et al. explored the relationship between childhood abuse and household dysfunction and many of the leading causes of death in adults. In that study, categories of childhood abuse included psychological abuse, physical abuse, or sexual abuse. Categories of household dysfunction during childhood included exposure to substance abuse, mental illness, violent treatment of a mother or stepmother, or criminal behavior in the household [2].

That study had several notable findings. First, it highlighted the prevalence of trauma. Conducted from 1995 to 1997 with 
two waves of data collection from over 17,000 Health Maintenance Organization members from Southern California receiving physical exams and completing confidential surveys, almost two thirds of participants reported at least one ACE while more than one in five participants reported having three or more ACEs [2]. Follow-up studies, including one through the CDC Behavioral Risk Factor Surveillance System BRFSS, showed similar results after collecting telephone surveys of 400,000 US adults each year [8]. In data from 2009 to 2014, again almost two thirds of participants reported at least one ACE while more than one in five participants reported having three or more ACEs [8].

Second, the landmark 1998 ACE study documented the significant impact of trauma. With an increased number of ACEs, there was an increased risk of negative health and well-being across the life course, including suicide attempts, depression, ischemic heart disease, liver disease, chronic obstructive pulmonary disease, smoking, early initiation of smoking, alcohol and alcohol use, illicit drug use, sexually transmitted diseases, financial stress, poor work performance, poor academic achievement, adolescent pregnancy, unintended pregnancies, fetal death, risk for sexual violence, and health-related quality of life. This effect was noted to have a graded dose-response relationship, with increased exposure to the stressor of ACEs leading to an increased intensity in this poor outcome. Individuals with four or more categories of ACEs had 4 to 12 times increased risk for suicide attempts, depression, alcoholism, and drug abuse compared to individuals who reported no ACEs. The influences throughout the lifespan of adverse child experiences could lead to social, emotional, and cognitive impairment; adoption of health-risk behaviors, disease, disability, and social problems and early death [2]. Later work by the National Comorbidity Survey Replication in 2001 explored questions of age of onset and the comorbidity of lifetime prevalence of psychiatric disorders including posttraumatic stress disorder, substance use disorders, and mood disorders [9].

Additional studies have noted the high prevalence of trauma. A recent study found that more than $70 \%$ of adults worldwide have experienced a traumatic event in their lifetime [10]. In the USA, physical and sexual assaults are the most frequently reported traumatic events at 52\% lifetime prevalence, followed by accidents or fires at $50 \%$ lifetime prevalence [10]. In the psychiatric population, trauma is highly prevalent and trauma histories can be complex, repeated, or ongoing [11]. PTSD is common in the USA and worldwide. Lifetime prevalence ranges from 1.3 to $12.2 \%$ depending on social background and country of residence [12]. The risk of PTSD is increased with traumatic brain injury, exposure to death, injury, torture, or bodily disfigurement, or exposure to an event that was unexpected, inescapable, or uncontrollable [13, 14]. PTSD may also present with other psychiatric diagnoses with more than 50\% of cases of PTSD having co-occurrence with mood, anxiety, or substance disorders [15].
Furthermore, the impact of trauma has neurobiological sequelae. Neural systems implicated in the pathophysiology of PTSD include the amygdala in fear learning; the amygdala, dorsal anterior cingulate cortex, insula in threat detection; frontoparietal connectivity in executive function and emotion regulation; as well as signaling between the medial prefrontal cortex and hippocampus in contextual processing [12]. Literature by Perez in 2017 showed inverse relationship between the burden of childhood abuse and left anterior insular volume, and between lifetime adverse events and left hippocampal volume [16]. In addition, a study by Protopopescu et al. demonstrated increased amygdalar activation with trauma-related versus neutral words in patients meeting criteria for PTSD [17]. Work by Liebenthal, Silbersweig, and Stern has explored the neural processing of language perception and emotional face cues, suggesting that an understanding of the underlying neurobiology is important for devising treatment approaches in alleviating emotional dysfunction [18]. There is, moreover, an impact on the chemical level, with neuroendocrine communication between the brain and body via hormonal pathways, impacting the neuroendocrine, autonomic, immune, and metabolic systems [19].

Within a trauma-informed care model, SAHMSA describes that "a program, organization, or system that is traumainformed realizes the widespread impact of trauma and understands potential paths for recovery; recognizes the signs and symptoms of trauma in clients, families, staff, and others involved with the system; responds by fully integrating knowledge about trauma into policies, procedures, and practices, and seeks to actively resist re-traumatization." Within this context, SAHMSA outlines six key principles of a traumainformed approach: (1) safety, (2) trustworthiness and transparency, (3) peer support, (4) collaboration and mutuality, (5) empowerment, voice, and choice, and (6) cultural, historical, and gender issues. SAHMSA sets forth four assumptions and six key principles to consider. The first assumption is that individuals at all levels of the organization have a basic realization of trauma and how it can affect broader constructs, such as families, groups, organizations, and communities, as well as individuals. Secondly, individuals in the organization must be able to recognize the signs of trauma, utilizing approaches such as screening, assessment, and workplace practices. Third, the organization responds by applying a traumainformed approach. Finally, the fourth assumption is that a trauma-informed approach seeks to resist re-traumatization of both patients and staff [20].

While trauma-informed care is a widely-used term and often utilized in social service settings, its application in daily practice of medicine and psychiatry is less well documented [21]. Much of the existing trauma-informed care information is located on websites of organizations within the USA, such as the Substance Abuse and Mental Health Services Administration (SAMHSA), Trauma-Informed Care Resources, and the 
National Association of State Mental Health Program Directors (NASMHPD) [22]. Existing literature for the implementation of trauma-informed care is predominately in the child and adolescent population. For example, a review by Bryson noted that many young people receiving psychiatric care in North American inpatient or residential settings have experienced various forms of emotional trauma and, furthermore, that the practices of these settings, such as seclusion and restraints, create a risk of retraumatization. That review explored articles that described trauma-informed care interventions in youth psychiatric or residential settings. However, even within this population the features that lead to successful implementation of trauma-informed care continue to be explored [23]. While most trauma-informed care is currently implemented by nonphysician staff, this paradigm shift needs physician engagement and leadership, with psychiatry as a field uniquely positioned for this role given our training in neurobiological underpinnings of the effect of trauma, our critical role in eliciting appropriate patient histories, our understanding of psychodynamic and psychotherapeutic principles, and the psychiatry consultation-liaison role of guiding multidisciplinary team meetings and challenging interactions in patient care [24].

\section{Trauma-Informed Care in Psychiatry Education}

Within the medical specialties, psychiatry residents are especially faced with the task of identifying and responding to patient histories, including that of trauma in various forms [23]. During training, psychiatry residents frequently have contact with patients and families with trauma histories, ranging from emergency room assessments through long-term recovery treatment [11]. In addition, psychiatry training includes significant work with patients who have substance use disorders and a majority of these individuals also have co-morbid traumatic exposures [25].

In addition to trauma history in the psychiatric population, trauma-informed care is especially well-suited to psychiatry given the intersection in psychiatry between a humanistic approach and neurobiological underpinnings of the field. Despite the ubiquitous nature of trauma, current graduate medical education requirements for psychiatry from the Accreditation Council for Graduate Medical Education (ACGME) do not outline specific standardized guidance for the teaching of trauma or trauma-informed care. A search of the document for the specific word "trauma" yields only a reference to traumatic brain injury. The requirements do note that residents must demonstrate competence in "recognizing and appropriately responding to family violence (e.g., child, partner, and elder physical, emotional, and sexual abuse and neglect) and its effect on both victims and perpetrators." However, there is no specific guidance for how this teaching should be integrated into the residency education curriculum, such as a number of hours of training, teaching modalities for shaping this competence, or how this may be folded into a psychiatry curriculum [26]. Educational guidance around teaching this topic within psychiatry medical education remains sparse. Even though physicians indicate that they would benefit from dedicated training, substantial gaps remain in the education of ACEs and trauma-informed competence [1].

Included in the 2019 ACGME Common Program Requirements for Residency are core competencies of professionalism (including well-being and self-care as well as transitions of care), patient care and procedural skills, medical knowledge, practice-based learning and improvement, interpersonal and communication skills, and systems-based practice. Although these requirements are not specific about training content, we assert that trauma-informed care principles and practices are relevant to each of those domains, offering a valuable framework for engaging patients, colleagues, and ourselves in ways that optimize outcomes for all participants in the health care system [27].

\section{Existing Literature on Trauma Education in Psychiatry}

Literature search for the development of a trauma curriculum for psychiatry residents is slim. Notably, in a 2014 publication, Ferrell et al. describe that they used the words trauma, education, and psychiatry residents as search terms in PsycINFO, PubMed, and Cochrane databases for articles reporting curricula on trauma topics for psychiatry residents, and at that time found only one curriculum targeted to mental health professionals and no studies evaluating the teaching of trauma curriculum [28].

Ferrell described the development of a 12-week course administered to 13 third-year psychiatry residents as part of didactics scheduling at Baylor College of Medicine. In this model, classes covered a wide variety of related topics, including childhood trauma, refugee trauma, survivors of torture, intimate partner violence, military sexual trauma, and natural disaster, and included techniques in therapy, role-playing activities, and pre- and post-course survey. However, even after the course, there was no change in the proportion of residents who felt comfortable assessing for trauma [28].

To help foster education for less prevalent local populations, literature by Pantziaras suggests that virtual patient systems could be utilized to improve confidence in providing transcultural clinical care for traumatized refugee patients [29]. Within this study, results explored included overall confidence in providing care for this population, as well as in specific domains of clinical care. These clusters included the ability to identify and evaluate trauma-related diagnoses and disability, to treat and manage trauma-related diagnoses and 
disability, to treat victims of torture, and to adapt work to different cultures via elements of intercultural communication [29].

Within broader medical training of physicians, literature by Goldstein explored the gap in trauma-informed care at the medical school level, via a sample of 20 medical students from the Summer Institute on Race and Health at the University of California, Davis. This study delivered a course including lectures, discussions, and practice, in two-hour modules over 3 days, with a post-training questionnaire on trauma-informed medical care. Results showed that this course increased the ability of participants to recognize clinical manifestations of adverse childhood experience (ACE) exposure in adult patients. That survey utilized five open-ended questions about the impact of the trauma training in medical school curriculum. While the course did provide education around asking about ACEs and responding to trauma disclosures, some participants also noted that one-time training as an elective or adjunct to a core curriculum was not sufficient to master trauma-informed medical care [1].

Additional literature on trauma-informed care in psychiatry is slim and from international rather than US-based findings. For example, work by Osman conducted an online survey of psychiatrists and primary care physicians from 17 Arab countries between September 2011 and June 2012 to explore trauma-informed care in that setting. That study highlighted the need to develop awareness and training programs in those Middle Eastern communities to better identify and treat trauma-related health and mental health issues [30]. A recent article by Isobel in 2016 explored the current state in Australia medical practice and emphasized a need for psychiatry to view trauma-informed care as a significant and urgent paradigm shift [31]. Work in the USA in 2017 by Beckett did highlight that embedding trauma-informed care principles in acute psychiatric inpatient ward practice is important given the impact of trauma, but that this change has been slow to occur [32].

\section{Applicability to Resiliency}

The topic of trauma within medical education also ties to the topic of resilience and the resiliency of the providers themselves. Resilience refers to the ability to recover from a stressful event, to adapt to change, and to persist in the face of adversity [1]. Psychiatry attendings and residents, as well as peers in other medical specialties, frequently work with trauma survivors. At the same time, these providers may themselves have been exposed to prior personal traumas or more recently to traumatic events that occur as part of their daily work. Organizational factors that contribute to high stress and potentially high staff turnover include high caseloads of patients who have experienced trauma as well as not providing trauma-informed care training and supervision, or failing to acknowledge the reality of secondary traumatization [4]. The interaction with patients with trauma histories may place residents, psychiatrists, and providers at risk for trauma themselves. For example, literature describes that professionals who conduct therapeutic work with trauma victims may experience secondary traumatic stress (STS) from repeatedly hearing the details of traumatic events that other individuals have experienced [33]. Similarly, literature notes that the characteristics that attract individuals into psychiatry and the caring professions make these same individuals vulnerable to compassion fatigue, defined as the caregiver's reduced capacity for and interest in bearing the suffering of others. In psychiatrists, the exposure to patients can increase the risk of vicarious trauma [34].

In this context, psychiatry as a field is well positioned for a leadership role in trauma-informed care, considering the organizational impact of these initiatives and helping to provide training across healthcare systems to increase resiliency among providers. For example, the Brigham Health Trauma Informed Care Initiative is increasingly recognized at the institutional level to be at the forefront of trauma-informed care (TIC) to optimize clinical care, to better care for patients and staff.

The need for psychiatry resident education on this topic is twofold. First, residency training provides the educational venue for residents to learn this material, for residents to join trauma-informed care activities, and for senior staff to model and guide trainees on trauma-informed care principles in practice. Second, residency training comes rife with challenging cases, exposure to traumas and death, and bearing witness to emotional narratives, all in the context of residents also undergoing personal and professional life changes. Psychiatry resident education on trauma and trauma-informed care provides an outstanding backdrop to also discuss the source of and strategies for resilience of both patients and providers themselves.

Resilience is a term increasingly referenced by medical education and the popular press, and even contemporary business models have begun to explore the connection between trauma and resiliency. Literature in the Harvard Business Review describes a module on post-traumatic growth that can be applied to business executives facing failure and applied to the psychological fitness of soldiers. This module encourages participants to understand the response to trauma, including shattered beliefs about the self, others, and the future, to reduce anxiety through techniques to control intrusive thoughts and to engage in constructive self-disclosure to avoid the physical and psychological symptoms of unresolved trauma. That approach helps to create a narrative in which trauma is seen for the personal strength and appreciation that it elicited, and encourages participants to articulate life principles to craft a new identity [35]. Harvard Business Review also describes a Master Resilience Training, patterned after the Penn 
Resiliency Program that helps participants build mental toughness, by exploring one's beliefs about adversity, exploring thinking traps such as overgeneralizing based upon a single action and minimizing catastrophic thinking. This training thereby helps participants explore and dispel unrealistic believes about adversity [35].

In the Goldstein study, students also completed the ACE Study and Resilience Questionnaires to assess the impact of ACEs and resilience on their lives. As part of the results of this literature, students pointed to the need for an organizational culture utilizing trauma-informed practices that are supportive of staff, providers, and patients [1].

\section{Call to Action}

This article highlights trauma-informed care as a critically needed component of psychiatry residency education curriculums. Psychiatry as a field is well positioned to be a leader in trauma-informed care (TIC), deepening and broadening its scope to incorporate an ever-evolving neurobiological understanding of the impacts of stressful exposures to research and clinical practice. In addition to encountering the high prevalence of trauma, psychiatry is aligned to provide both humanistic connection in patient encounters as well as integrate the neuroscience unpinning of trauma and its biological sequelae. This increased focus on trauma-informed care can tie to broader hospital organizational initiatives, such as the Brigham Health Trauma Informed Care Initiative traumainformed care initiative, as well as to provide discussions and teachings on resiliency to benefit patients as well as staff and providers.

Existing models for designing education in psychiatry residency for trauma and trauma-informed care is astonishingly slim in contemporary literature. Given the lack of existing guidelines in contemporary literature regarding the design of trauma-informed care education for psychiatry trainees, additional work is needed to study the efficacy of specific teaching interventions and to incorporate feedback to iteratively improve the educational offerings.

Leveraging the concepts set forth in this article, these authors would recommend a proposed residency education curriculum of incorporating trauma-informed care topics into didactic sessions, providing more formal teaching on the tenants of trauma-informed care, including case-based education on trauma-informed care and considering the role of simulationbased exercises to practice these techniques. This education would best be incorporated into both didactics and supervision in order to build upon educational teaching and to reinforce these trauma-informed tenets across training years. Ideally, this education would be integrated, exploring both humanism with patients and colleagues as well as the neurobiological effects of stress, trauma, and resilience, in order to optimize outcomes for patients and support the psychiatrists who strive to care for them. Building upon medical school training, this educational module would address trauma-informed care implications in treating a full spectrum of psychiatric disorders. This curriculum would, therefore, position psychiatry residents as potential leaders in trauma-informed care and interactive efforts to generate an evolving understanding of trauma and its impact among all stakeholders in the healthcare system.

Acknowledgements David A. Silbersweig, MD and Abi Warren, BA.

\section{Compliance with Ethical Standards}

Disclosure On behalf of all authors, the corresponding author states that there is no conflict of interest.

Ethical Considerations No human participants were involved.

Publisher's Note Springer Nature remains neutral with regard to jurisdictional claims in published maps and institutional affiliations.

\section{References}

1. Goldstein E, Murray-Garcia J, Sciolla AF, Topitzes J. Medical students' perspectives on trauma-informed care training. Perm J. 2018;22:17-126.

2. Felitti VJ, Anda RF, Nordenberg D, Williamson DF, Apitz AM, Edwards V, et al. Relationship of childhood abuse and household dysfunction to many of the leading causes of death in adults. Am J Prev Med. 1998;14(4):245-58.

3. Campbell JA, Walker RJ, Egede LE. Associations between adverse childhood experiences, high-risk behaviors, and morbidity in adulthood. Am J Prev Med. 2016;50(3):344-52.

4. McEwen BS. Allostasis and the epigenetics of brain and body health over the life course: the brain on stress. JAMA Psychiatry. 2017;74(6):551-2.

5. Substance Abuse and Mental Health Services Administration (SAMHSA). Trauma-informed care in behavior health services. Treatment improvement protocol (tip) series, No. 57. Center for substance abuse treatment (US) Rockville (MD). 2014. Chapter 2 Building a Trauma-informed Workforce.

6. Substance Abuse and Mental Health Services Administration (SAMHSA). Trauma-informed approach and trauma-specific interventions. https://www.samhsa.gov/nctic/trauma-interventions

7. Kilpatrick DG, Resnick HS, Milanak ME, Miller MW, Keyes KM, Friedman MF. National Estimates of exposure to traumatic events and PTSD prevalence using DSM-IV and DSM-5 criteria. J Trauma Stress. 2013;26(5):537-47.

8. Centers for Disease Contorl and Prevention (CDC). About behavioral risk factor surveillance system ACE data. https://www.cdc. gov/violenceprevention/acestudy/ace_brfss.html

9. Kessler RC, Berglund P, Demler O, Jin R, Merikangas KR, Walters EE. Lifetime prevalence and age-of-onset distributions of DSM-IV disorders in the National Comorbidity Survey replication. Arch Gen Psychiatry. 2005;62:593-602.

10. Benjet C, Bromet E, Karam EG, Kessler RC, McLaughlin KA, Ruscio AM, et al. The epidemiology of traumatic event exposure worldwide: results from the world mental health survey consortium. Psychol Med. 2016;46:327-43. 
11. Schneeberger AR, Muenzenmaier K, Abrams M, Antar L, Leon SR, Ruberman L, et al. Comprehensive trauma training curriculum for psychiatry residents. Acad Psychiatry. 2012;36(2):136-7.

12. Shalev A, Liberzon I, Marmar C. Post-traumatic stress disorder. N Engl J Med. 2017;376:2459-69.

13. Yurgil KA, Barkauskas DA, Vasterling JJ, Nievergelt CM, Larson GE, Schork NJ, Litz BT, Nash WP, Baker DG, Marine Resiliency Study Team. Association between traumatic brain injury and risk of posttraumatic stress disorder in active-duty marines. JAMA Psychiatry 2014; 71: 149-157.

14. Delahanty DL, Nugent NR, Christopher NC, Walsh M. Initial urinary epinephrine and cortisol levels predict acute PTSD symptoms in child trauma victims. Psychoneuroendocrinology. 2005;30:121-8.

15. Pietrzak RH, Goldstein RB, Southwick SM, Grant BF. Prevalence and axis I comorbidity of full and partial posttraumatic stress disorder in the United States: results from wave 2 of the National Epidemiologic Survey on alcohol and related conditions. J Anxiety Disord. 2011;25:456-65.

16. Perez DL, Matin N, Barsky A, Costumero-Ramos V, Makaretz SJ, Young SS, et al. Cingulo-insular structural alterations associated with psychogenic symptoms, childhood abuse and PTSD in functional neurological disorders. J Neurol Neurosurg Psychiatry. 2017;88(6):491-7.

17. Protopopescu X, Pan H, Tuescher O, Cloitre M, Goldstein M, Engelien W, et al. Differential time courses and specificity and amygdala activity in posttraumatic stress disorder subjects and normal control subjects. Biol Psychiatry. 2005;57(5):464-73.

18. Liebenthal E, Silbersweig DA, Stern E. The language, tone, and prosody of emotions: neural substrates and dynamics of spokenword emotion perception. Front Neurosci. 2016;10:506.

19. McEwen BS, Gray JD, Nasca C. Redefining neuroendocrinology: stress, sex and cognitive and emotional regulation. J Endocrinol. 2015;226(2):T67-83.

20. Substance Abuse and Mental Health Services Administration (SAMHSA)'s trauma and justice strategic initiative. SAMHSA's concept of trauma and guidance for a trauma-informed approach. July 2014. http://www.traumainformedcareproject.org/resources/ SAMHSA\%20TIC.pdf

21. Raja S, Hasnain M, Hoersch M, Gove-Yin S, Rajagopalan C. Trauma informed care in medicine: current knowledge and future research directions. Fam Community Health. 2015;38(3):216-26.

22. Muskett C. Trauma-informed care in inpatient mental health settings: a review of the literature. Int J Ment Health Nurs. 2014;23(1): $51-9$.
23. Bryson S, Gauvin E, Jamieson A, et al. What are effective strategies for implementing trauma-informed care in youth inpatient psychiatric and residential treatment settings? A realist systematic review. Int J Mental Health Syst. 2017;11(36):1-16.

24. Levy-Carrick NC, Lewis-O'Connor A, Rittenberg E, Manosalvas K, Stoklosa HM, Silbersweig D. Promoting health equity through trauma informed care: critical role for physicians in policy and program development. Fam Community Health. 2019;42(2). https://doi.org/10.1097/FCH.0000000000000214.

25. Sofuoglu M, Rosenheck R, Petrakis I. Pharmacological treatment of comorbid PTSD and substance use disorder: recent progress. Addict Behav. 2014;39(2):428-33.

26. Accreditation Council for Graduate Medical Education. ACGME program requirements for graduate medical education in psychiatry. July 1, 2017. Line IV.A.5.A).(1). (b).(xiii). http://www.acgme.org/ Portals/0/PFAssets/ProgramRequirements/400_psychiatry_201707-01.pdf?ver=2017-05-25-083803-023.

27. Accreditation Council for Graduate Medical Education. ACGME common program requirements (residency). Approved June 10, 2018. Effective July 1, 2019. https://www.acgme.org/Portals/0/ PFAssets/ProgramRequirements/CPRResidency2019.pdf. 18-22.

28. Ferrell NJ, Melton B, Banu S, Coverdale J, Valdez MR. The development and evaluation of a trauma curriculum for psychiatry residents. Acad Psychiatry. 2014;38:611-4.

29. Pantziaras I, Fors U, Ekblad S. Innovative training with virtual patients in transcultural psychiatry: the impact on resident psychiatrists' confidence. PLoS One. 2015;10(3):e0119754.

30. Osman OT, Nasir L, Mollica RF, et al. Trauma-informed care survey of psychiatrist and primary care physicians in the Middle East. Prim Care Companion CNS Disord. 2017;19(5):e1-8.

31. Isobel S. Trauma informed care: a radical shift or basic good practice? Aust Psychiatry. 2016;24(6):589-91.

32. Beckett P, Holmes D, Phipps M, Patton D, Molloy L. Traumainformed care and practice: practice improvement strategies in an inpatient mental health ward. J Psychosoc Nurs Ment Health Serv. 2017;55(10):34-8.

33. Hensel JM, Ruiz C, Finney C, Dewa CS. Meta-analysis of risk factors for secondary traumatic stress in therapeutic work with trauma victims. J Trauma Stress. 2015;28(2):83-91.

34. Franza F, Del Buono G, Pellegrino F. Psychiatric caregiver stress: clinical implications of compassion. fatigue. 2015;27(Suppl 1): S321-7.

35. Seligman MEP. Building resilience. Harvard Business Review. April 2011. 